\title{
Lewin's homogenization formula revisited for nanocomposite materials
}

\author{
Tom G. Mackay \\ School of Mathematics and Maxwell Institute for Mathematical Sciences, University of \\ Edinburgh, Edinburgh EH9 3JZ, Scotland, United Kingdom \\ T.Mackay@ed.ac.uk
}

\begin{abstract}
The applicability of Lewin's homogenization formula is restricted to composite materials wherein: (a) the inclusions are small relative to wavelength in the host material and the inclusion material; (b) the real parts of the permittivities (and/or the permeabilities) of the host and inclusion materials have the same sign, for weakly nondissipative materials; and (c) the volume fraction of the inclusion material is less than approximately 0.3 .
\end{abstract}

Keywords: extended Maxwell Garnett, quasistatic limit, metamaterial

\section{INTRODUCTION}

Homogenization formalisms, such as the well-established Maxwell Garnett formalism [1], can provide useful estimates of the constitutive parameters of homogenized composite materials (HCMs) [2-4]. However, the implementation of these formalisms is appropriate only in certain circumstances.

In 1946 Lewin presented an extended version of the Maxwell Garnett homogenization formalism [5]. Lewin's formula has recently attracted attention in the context of HCMs which support negative-phase-velocity propagation of plane waves and can therefore refract negatively [6-10]. The constituents of the composite material considered by Lewin are: (i) an isotropic dielectric-magnetic host material with relative permittivity $\epsilon_{1}$ and relative permeability $\mu_{1}$; and (ii) an isotropic dielectric-magnetic inclusion material with relative permittivity $\epsilon_{2}$ and relative permeability $\mu_{2}$. The inclusion material has the form of spherical particles, each of radius $a$, arranged on a cubic lattice with $f$ denoting the volume fraction of the inclusion material; Lewin's homogenization formula has also been applied to composites with randomly distributed inclusions [9]. The dimensionless parameter

$$
\theta=\frac{2 \pi a \sqrt{\epsilon_{2} \mu_{2}}}{\lambda}
$$

gauges the size of the inclusions relative to characteristic electromagnetic length-scales in the inclusion material. Herein, $\lambda$ is the free-space wavelength. Lewin's estimates of the relative permittivity and relative permeability of the HCM are [5]

$$
\kappa_{\mathrm{HCM}}=\kappa_{1}\left[1+3 f\left(\frac{\kappa_{2} F(\theta)+2 \kappa_{1}}{\kappa_{2} F(\theta)-\epsilon_{1}}-f\right)^{-1}\right], \quad(\kappa=\epsilon, \mu),
$$

respectively, wherein the function

$$
F(\theta)=\frac{2(\sin \theta-\theta \cos \theta)}{\left(\theta^{2}-1\right) \sin \theta+\theta \cos \theta} .
$$

Alternative representations for Lewin's estimates of $\epsilon_{\mathrm{HCM}}$ and $\mu_{\mathrm{HCM}}$ can be provided in terms of spherical Bessel and spherical Hankel functions [7]. 
Instead of the usual Mossotti-Clausius expression for the polarizability of an isotropic dielectric sphere embedded in an isotropic dielectric host material [11, Sec. 4.5] and its analog for the magnetizability of an isotropic magnetic sphere embedded in an isotropic magnetic host material, Lewin [5] used expressions obtained from the first two coefficients (often designated as $a_{1}$ and $b_{1}$ ) of the Lorenz-Mie-Debye solution for the scattering of a plane wave by an isotropic dielectric-magnetic sphere embedded in an isotropic dielectric-magnetic host material [12, Sec 4.3]. That practice is fine, but it does not provide a license to remove the restriction on the magnitude of $\theta$ when implementing the Maxwell Garnett and other similar formalisms.

Noting that homogenization formulas can be applicable to composite materials containing nanosized inclusions, in this letter crucial limitations of Lewin's formula are identified which have not been respected in recent studies [6-10].

\section{LIMITATIONS}

There are three limitations on Lewin's homogenization formula as follows.

(a) In Lewin's analysis [5], the inclusions were taken to be small relative to wavelength in the host material but not in the inclusion material; i.e., $\left|a \sqrt{\epsilon_{1} \mu_{1}}\right| \ll \lambda$ but not $|\theta / 2 \pi| \ll$ 1.

Homogenization of constitutive parameters is a valid concept if the spatial variations of the electric and magnetic fields are slow everywhere in the actual composite material. The condition $\left|a \sqrt{\epsilon_{1} \mu_{1}}\right| \ll \lambda$ ensures that the exciting fields are virtually uniform over the region occupied by an inclusion. The condition $|\theta / 2 \pi| \ll 1$ ensures that the fields induced inside the inclusion are virtually uniform as well.

Van de Hulst [13, Sec. 6.4] has actually stipulated even stronger conditions: $\left|2 \pi a \sqrt{\epsilon_{1} \mu_{1}}\right|$ $\ll \lambda$ and $|\theta| \ll 1$. He has pointed out that, if the condition $\left|2 \pi a \sqrt{\epsilon_{1} \mu_{1}}\right| \ll \lambda$ is true but the condition $|\theta| \ll 1$ is not, then resonances will arise inside the inclusion, a multipolar (and not simply dipolar) representation of the inclusion will have to be considered, and the spatial variations of the fields in the HCM will then be rapid in the region occupied by an inclusion. Accordingly, suitable bounds which must be satisfied for homogenization are $\left|a \sqrt{\epsilon_{1} \mu_{1}}\right| \leq \lambda / 10$ and $|\theta / 2 \pi| \leq 1 / 10$. These bounds on the relative sizes of the inclusions apply generally to homogenization formalisms, such as the Maxwell Garnett formalism and formulas based on it (e.g., Lewin's formula), and the Bruggeman formalism which arises from the strong-property-fluctuation theory [14].

(b) If

$$
\operatorname{Re}\left\{\epsilon_{1}\right\} \operatorname{Re}\left\{\epsilon_{2}\right\}<0 \quad \text { and }\left|\operatorname{Im}\left\{\epsilon_{\ell}\right\}\right| \ll\left|\operatorname{Re}\left\{\epsilon_{\ell}\right\}\right|, \quad(\ell=1,2)
$$

and/or

$$
\operatorname{Re}\left\{\mu_{1}\right\} \operatorname{Re}\left\{\mu_{2}\right\}<0 \quad \text { and }\left|\operatorname{Im}\left\{\mu_{\ell}\right\}\right| \ll\left|\operatorname{Re}\left\{\mu_{\ell}\right\}\right|, \quad(\ell=1,2),
$$

then it is inappropriate to apply the Maxwell Garnett formalism-or formulas such as Lewin's formula which represent inclusion-size adjustments to the Maxwell Garnett formalism. This is because the estimated constitutive parameters of the HCM exhibit strong resonances with respect to the volume fraction $f$, but those resonances are not physically plausible [15]. Indeed, this limitation applies also to the Bruggeman homogenization formalism [15] and the Bergman-Milton bounds on the constitutive parameters of HCMs [16]. The conditions (4) may be satisfied at optical frequencies when the inclusions are made from certain noble metals, such as gold or silver, and the host material is an insulator [17]. 
(c) A major drawback of the Maxwell Garnett formalism - which also afflicts formulas such as Lewin's formula that are essentially based on the Maxwell Garnett formalism is its failure to predict a percolation threshold. In contrast, the Bruggeman homogenization formalism - in which the inclusion material and the host material have exactly the same footing — predicts a threshold at $f=1 / 3$ [18, Sec. 8.3]. Accordingly, it is appropriate to use the Maxwell Garnett formalism at $f \lesssim 0.3$ only (unlike the Bruggeman formalism which may be applied for $0<f<1$ ).

\section{CONCLUDING REMARKS}

While conventional homogenization formalisms are undoubtedly of great benefit to material designers in certain circumstances, they cannot be implemented without due regard to their limitations. This point is particularly relevant in the context of HCMs as metamaterials, which often involve unconventional ranges of the constitutive parameters. The applicability of Lewin's formula is restricted to regimes where:

(a) the inclusions are small relative to wavelength in the host material and in the inclusion material;

(b) the conditions (4) and (5) are not satisfied; and

(c) the volume fraction $f \lesssim 0.3$.

\section{References}

[1] J. C. Maxwell Garnett, "Colours in metal glasses and in metallic films," Phil. Trans. R. Soc. Lond. A 203, 385-420 (1904) [doi:10.1098/rspl.1904.0058].

[2] A. Lakhtakia (Ed.), Selected Papers on Linear Optical Composite Materials, SPIE Optical Engineering Press, Bellingham, WA, USA (1996).

[3] A. Lakhtakia, "On direct and indirect scattering approaches for the homogenization of particulate composites," Microw. Opt. Technol. Lett. 25, 53-56 (2000) [doi:10.1002/(SICI)1098-2760(20000405)25:1<53::AID-MOP16>3.0.CO;2-5]

[4] C. Brosseau, "Modelling and simulation of dielectric heterostructures: a physical survey from an historical perspective," J. Phys. D: Appl. Phys. 39, 1277-1294 (2006) [doi: 10.1088/0022-3727/39/7/S02].

[5] L. Lewin, "The electrical constants of a material loaded with spherical particles," Proc. IEE 94, 65-68 (1947).

[6] C. L. Holloway, E. F. Kuester, J. Baker-Jarvis, and P. Kabos, "A double negative (DNG) composite medium composed of magnetodielectric spherical particles embedded in a matrix," IEEE Trans. Antennas Propagat. 51, 2596-2603 (2003) [doi:10.1109/TAP.2003.817563].

[7] V. Yannopapas and A. Moroz, "Negative refractive index metamaterials from inherently non-magnetic materials for deep infrared to terahertz frequency ranges," J. Phys.: Condens. Matter 17, 3717-3734 (2005) [doi:10.1088/0953-8984/17/25/002].

[8] M. S. Wheeler, J. S. Aitchison, and M. Mojahedi, "Coated nonmagnetic spheres with a negative index of refraction at infrared frequencies," Phys. Rev. B 73, 045105 (2006) [doi:10.1103/PhysRevB.73.045105].

[9] A.-G. Kussow, A. Akyurtlu, and N. Angkawisittpan, "Optically isotropic negative index of refraction metamaterial," phys. stat. sol. (b) 245, 992-997 (2008) [doi:10.1002/pssb.200743377].

[10] C.-W. Qiu and L. Gao, "Resonant light scattering by small coated nonmagnetic spheres: magnetic resonances, negative refraction, and prediction," J. Opt. Soc. Am. B 25, 1728 1737 (2008) [doi:10.1364/JOSAB.24.001225]. 
[11] J. D. Jackson, Classical Electrodynamics, 3rd ed., Wiley, Hoboken, NJ, USA (1999).

[12] C. F. Bohren and D. R. Huffman, Absorption and Scattering of Light by Small Particles, Wiley, New York, NY, USA (1983).

[13] H. C. van de Hulst, Light Scattering by Small Particles, Dover, New York, NY, USA (1981).

[14] T. G. Mackay, "On extended homogenization formalisms for nanocomposites," $J$. Nanophoton. 2, 021850 (2008) [doi:10.1117/1.2982931].

[15] T. G. Mackay and A. Lakhtakia, "A limitation of the Bruggeman formalism for homogenization," Opt. Commun. 234, 35-42 (2004) [doi:10.1016/j.optcom.2004.02.007].

[16] A. J. Duncan, T. G. Mackay, and A. Lakhtakia, "On the Bergman-Milton bounds for the homogenization of dielectric composite materials," Opt. Commun. 271, 470-474 (2007) [doi:10.1016/j.optcom.2006.10.056].

[17] T. G. Mackay, "On the effective permittivity of silver-insulator nanocomposites," $J$. Nanophoton. 1, 019501 (2007) [doi:10.1117/1.2472372].

[18] L. Ward, The Optical Constants of Bulk Materials and Films, 2nd ed., Institute of Physics, Bristol, UK (1994).

Tom G. Mackay undertook his PhD in electromagnetic theory at the Department of Mathematics, University of Glasgow, under the supervision of the late Werner S. Weiglhofer. In 2001 he moved to the University of Edinburgh, where he is now a reader in the School of Mathematics. His current research interests include homogenization, complex materials, metamaterials, and sculptured thin films. For further details see http : //www.maths.ed.ac.uk/ tgm. 